\title{
Mars Ascent Vehicle needs a Sustained Development Effort, Regardless of Sample Return Mission Timelines
}

\author{
John Whitehead
}

$530-758-8115$

Consultant

john.whitehead@alumni.caltech.edu

A white paper submitted for the Decadal Survey on Planetary Science and Astrobiology

2020 June 7 (first of three by this author)

Revised with minor corrections, clarifications, and some additions

Resubmitted on 2020 September 11 


\section{$\underline{\text { Key Points }}$}

The main point of this white paper is that that developing a Mars Ascent Vehicle (MAV) is a huge technology challenge that needs a sustained rocket engineering effort, even if plans for Mars Sample Return (MSR) happen to slip further into the future. A MAV is a unique new thing that might not be ready to support the currently envisioned timeline for MSR. Even if a MAV is not ready for a sample return lander to depart from Earth in 2026 or 2028, building and testing prototype MAVs will need a steady ongoing effort, in order for MSR to ultimately succeed.

A serious effort toward MAV development has been postponed for decades, essentially waiting for MSR plans to be in place. However, there are reasons to be concerned that the order of events might need to be reversed. Namely, MSR planning may need to wait for successful MAV development. The reason is that it may be overoptimistic to assume that a MAV engineering effort will fit into predetermined budgets and schedules for MSR.

The MAV is going to be the most amazing little rocket ever built. Considering the large velocity change it needs to deliver $(\sim 4,000 \mathrm{~m} / \mathrm{s})$ and its high thrust-to-mass ratio (on the order of 10 $\mathrm{m} / \mathrm{s}^{\wedge} 2$ ), the MAV needs to be a miniature launch vehicle, not a spacecraft in the usual sense. The MAV will be far more capable than any past or present spacecraft or missile of its small size. Ascending from Mars to orbit is entirely different from launching off the moon, due to the far higher speed and acceleration requirements, along with atmospheric drag [1].

If a MAV could be very small ( $<100 \mathrm{~kg}$ ), as was hoped circa 1998, then MSR likely could have been done by now. After many MAV design studies, separated by multi-year periods of relative inactivity, no one really knows how small a MAV can be, how long it will take to bring to fruition, or how much payload mass can realistically be expected.

The difficulty of making a smaller MAV is the primary reason why returning samples is presently envisioned as a separate mission from science rovers. Nevertheless, it remains unproven that a MAV can be small enough to send to Mars during the coming decade, along with its Mars launch support equipment and environmental survival aids such as temperature control. The present delivery capability for a Mars lander is roughly one metric ton, as exemplified by the Mars Science Laboratory (MSL, "Curiosity") and Mars 2020 ("Perseverance").

No one really even knows what kind of rocket technology will turn out to make a MAV that is functional, reliable, and affordable. Different engineering design studies have reached a variety of conclusions, because assumptions are necessarily somewhat arbitrary and hopeful in the absence of sufficient experience building and testing miniature launch vehicles. One strong indication of top-level uncertainty is the major redirection of MAV research in 2019. Early in 2018, a notional single-stage, hybrid propellant (solid fuel + liquid oxidizer) MAV design was presented to MEPAG as the likely solution [2], and it subsequently received a favorable mention 
in the Decadal midterm review [3]. However, as of early 2020, a two-stage solid propellant MAV design is being favored [4,5]. Another future redirection cannot be ruled out.

A huge part of the MAV development problem is that creative engineering work is likely necessary to realize unusually light weights for propulsion components, but funding for MAV efforts has come with limited timelines and optimism that intellectual leaps are not needed. No one really knows to what extent MAV development needs to include trial-and-error building and testing, as opposed to only needing a schedule-budget-design-build-fly effort.

To restate the main key point, MSR is more likely to ultimately happen if MAV development continues steadily, as opposed to going dormant in the event that plans for MSR are postponed. For the previous decadal survey, NASA technology leaders wrote that MAV flight testing (in Earth's thin upper atmosphere) should occur 4-5 years before Earth departure, enabled by component technology readiness 7-8 years in advance [6]. Based on this 2009 assessment, MAV team efforts to meet the 2026 date will be heroic. A recent MSR program schedule shows a MAV development timeline without explicitly indicating flight testing [7].

\section{Further Discussion of the Key Points}

A MAV will be something entirely new, so it should not be surprising if the MAV team will need to include a significant amount of new or expanded technical expertise. Considering the uniqueness of a MAV, in the event of a lapse in the effort, there may not be another program or a customer to pay for continuity of the specialized expertise. One hypothetical possibility is that a MAV would be similar to the upper stage of a very small Earth launch vehicle. While such a tiny launch vehicle could put individual cubesats in Earth orbit, investment is uncertain because economies of scale work against business sustainability [8].

Any commercial effort, to develop the kind of rocket technology needed for a MAV, is unlikely to find a sustainable market. For the sake of contrast, consider that imaging sensor expertise applies to essentially all planetary science missions, while advances in the underlying technology are associated with huge markets for digital cameras. MAV propulsion technology might find applications to other science spacecraft, perhaps lunar surface access, but lunar landing and return do not need rockets as special as a MAV [1].

Considering the potential need for trial-and-error MAV development, one shining example of such activity has been provided by SpaceX for Earth launch vehicle development. Amazing successes, along with whole new capabilities, have been made possible only by many unwanted setbacks in testing. It might be wrong to assume in advance that the MAV team will be able to make the components as lightweight as needed, without similar trial-and-error testing. 
The primary engineering challenge for a MAV is to make all the parts unusually lightweight relative to the propellant mass, in a final assembled configuration. Therefore, there is really no such thing as "having MAV technology in hand" without a complete MAV. The widely used metric for Technology Readiness Level (TRL scale) for example, does not directly recognize "lightweight enough to fly" versus "too heavy to fly."

Special efforts to reduce the mass of parts were highlighted in a paper about the smallest rocket to ever reach Earth orbit, the Japanese SS-520-5, in 2018 [9]. The latest MAV design papers do not emphasize such efforts, which will become essential in the near future $[10,11]$.

MAV technology is not known well enough to even select a particular propellant, or a particular number of stages, in advance. This statement is supported by the fact that decades of MAV design studies have reached a wide variety of conclusions. The primary reason for the uncertainty is the need for unusually high propulsive capability to be built into an unusually small rocket propelled vehicle.

A sustained effort toward MAV development is way overdue, after decades of interest in MSR with mostly only design studies for MAV concepts. The postponement is one reason why the Mars 2020 mission is one large rover, instead of potentially a smaller rover that carries a small MAV. No one really knows how small a MAV can be, or how big and heavy a MAV needs to be in order to be successful. Therefore, the Mars ascent payload size might need iteration.

In conclusion, the ultimate long-term science value of MSR strongly supports the value of an ongoing sustained MAV development program, regardless of actual MSR mission plans. Years of engineering development, with at least some iterative design and testing, including multiple flight tests, will most likely be needed to result in a mission-capable MAV.

\section{About the author}

Starting in the early 1990's, the author has followed the many sporadic efforts toward MAV development, and has participated professionally at various times in the past. His miniature propulsion research, initiated in 1988, was funded by the NASA Mars Program from 2005 to 2007 [12]. His decade of papers specific to Mars ascent included calculations to show how trajectories from Mars to orbit are intertwined with MAV propulsion design [13]. He was an invited presenter at the 2008 meeting referred to in Reference 6, the NASA Workshop on MSR Technologies at the Lunar and Planetary Institute.

He has made efforts to raise MAV awareness in the Mars community [14-16]. He has reached out to, and discussed the MAV problem with, Mars scientists at three institutions he has been associated with, UC Davis, Caltech, and Lawrence Livermore National Laboratory. He has also reached out to JPL managers and engineering leaders at other NASA centers, to raise awareness 
about the MAV challenge. Presently he advises U.S. Government officials on technical matters related to the goal of slowing missile technology proliferation around the world.

\section{References cited}

1. Whitehead, J.C., "Defining the Mars Ascent Problem for Sample Return," American Institute of Aeronautics and Astronautics, AIAA-2008-7768, Space 2008, San Diego, CA, September 2008. https://arc.aiaa.org/doi/abs/10.2514/6.2008-7768 https://arc.aiaa.org/doi/10.2514/6.2008-7768

2. Edwards, C., "Mars Sample Return Capability Development: Mars Ascent Vehicle and Mars On-Orbit Rendezvous," presented to the Mars Exploration Program Analysis Group (MEPAG), 20 February 2018.

https://mepag.jpl.nasa.gov/meeting/2018-02/04 Edwards MSRMidDecadal MEPAG VM1.pdf

3. Space Studies Board, National Academies of Sciences, Engineering, and Medicine "Vision and Voyages for Planetary Sciences in the Decade 2013-2022: A Midterm Review," The National Academies Press, Washington DC, 2018, ISBN 978-0-309-47933-2, p 5-13.

https://doi.org/10.17226/25186 or https://www.nap.edu/catalog/25186/visions-into-voyagesfor-planetary-science-in-the-decade-2013-2022

4. Muirhead, B., et al, "Mars Sample Return Mission Concept Status," 2020 IEEE Aerospace Conference, Big Sky, MT, March 2020. https://ieeexplore.ieee.org/document/9172609

5. Clark, S., "NASA narrows design for rocket to launch samples off of Mars," Spaceflight Now, 20 April 2020. https://spaceflightnow.com/2020/04/20/nasa-narrows-design-for-rocket-tolaunch-samples-off-of-mars/

6. Hayati, S., et al, "Strategic Technology Development for Future Mars Missions (2013-2022)," A white paper submitted to the National Research Council as input to the 2009 Planetary Decadal Survey, September 2009. https://mepag.jpl.nasa.gov/reports.cfm https://mepag.jpl.nasa.gov/reports/decadal/SamadAHayati.pdf

7. Gramling, J., "Mars Sample Return (MSR) Presentation to PAC," Presented to the Planetary Science Advisory Committee, August 2020.

https://science.nasa.gov/science-red/s3fs-public/atoms/files/07-GramlingMSRPAC\%2017Aug2020.pdf

8. Hunsaker, T., et al, "Nanosat Launch Vehicles: A Global Perspective and Business Case," American Institute of Aeronautics and Astronautics, AIAA-2013-5513, Space 2013, San Diego, CA, September 2013. https://arc.aiaa.org/doi/10.2514/6.2013-5513 
9. Inatani, Y., and Ohtsuka, H., "SS-520 Nano Satellite Launcher and its Flight Result," 32nd Annual Small Satellite Conference, SSC18-IX-03, August 2018.

https://digitalcommons.usu.edu/smallsat/2018/all2018/308/

10. Yaghoubi, D., and Schnell, A., "Mars Ascent Vehicle Hybrid Propulsion Configuration," 2020 IEEE Aerospace Conference, Big Sky, MT, March 2020.

https://ieeexplore.ieee.org/document/9172479

11. Yaghoubi, D., and Schnell, A., "Mars Ascent Vehicle Solid Propulsion Configuration," 2020 IEEE Aerospace Conference, Big Sky, MT, March 2020. https://ieeexplore.ieee.org/document/9172654

12. Whitehead, J.C., "Pump Fed Propulsion for Mars Ascent and Other Challenging Maneuvers," Proceedings of the NASA Science Technology Conference NSTC-2007, College Park, MD, June 2007, ISBN 0-9785223-3-8, Paper number D11P1.

http://citeseerx.ist.psu.edu/viewdoc/download?doi=10.1.1.692.2075\&rep=rep1\&type=pdf

13. Whitehead, J.C., "Trajectory Analysis and Staging Trades for Smaller Mars Ascent Vehicles," Journal of Spacecraft and Rockets, Vol. 42, No. 6, Nov-Dec 2005, pp 1039-1046.

https://arc.aiaa.org/doi/abs/10.2514/1.10680?journalCode=jsr

14. Whitehead, J.C., "Mars needs technology designed for sample return," Nature, Vol. 449, p 972, 25 October 2007. https://www.nature.com/articles/449972c

15. Whitehead, J.C., "A Perspective on Mars Ascent for Scientists," Concepts and Approaches for Mars Exploration, Lunar and Planetary Institute, Houston, TX, 12-14 June 2012, Abstract number 4290. https://www.lpi.usra.edu/meetings/marsconcepts2012/pdf/4290.pdf

16. Whitehead, J.C., "Mars Ascent Vehicle Needs Technology Development with a Focus on High Propellant Fractions," 2nd International Mars Sample Return Conference, Berlin, 25-27 April 2018, Abstract number 6035 (LPI contribution number 2071). https://www.Ipi.usra.edu/lpi/contribution docs/LPI-002071.pdf (scroll to page 168) https://www.hou.usra.edu/meetings/marssamplereturn2018/pdf/6035.pdf (quick link) 